\title{
Development of an Odd-Z-Projectile Reaction for Heavy Element Synthesis: ${ }^{208} \mathrm{~Pb}\left({ }^{64} \mathrm{Ni}, n\right){ }^{271} \mathrm{Ds}$ and ${ }^{208} \mathrm{~Pb}\left({ }^{65} \mathrm{Cu}, n\right){ }^{272} 111$
}

\author{
C. M. Folden III, ${ }^{1,2}$ K. E. Gregorich, ${ }^{1}$ Ch. E. Düllmann, ${ }^{1,2}$ H. Mahmud, ${ }^{1}$ G. K. Pang, ${ }^{1,2}$ J. M. \\ Schwantes, ${ }^{1,2}$ R. Sudowe, ${ }^{3}$ P. M. Zielinski, ${ }^{1,2}$ H. Nitsche, ${ }^{1,2}$ and D. C. Hoffman ${ }^{1,2}$
}

\footnotetext{
${ }^{1}$ Nuclear Science Division, Lawrence Berkeley National Laboratory, Berkeley, California 94720

${ }^{2}$ Department of Chemistry, University of California, Berkeley, California 94720

${ }^{3}$ Chemical Sciences Division, Lawrence Berkeley National Laboratory, Berkeley California
} 94720

Seven ${ }^{271}$ Ds decay chains were identified in the bombardment of ${ }^{208} \mathrm{~Pb}$ targets with 311.5- and 314.3-MeV ${ }^{64} \mathrm{Ni}$ projectiles using the Berkeley Gas-filled Separator. These data, combined with previous results, provide an excitation function for this reaction. From these results, an optimum energy of $321 \mathrm{MeV}$ was estimated for the production of ${ }^{272} 111$ in the new reaction ${ }^{208} \mathrm{~Pb}\left({ }^{65} \mathrm{Cu}, n\right)$. One decay chain was observed, resulting in a cross section of $1.7_{-1.4}^{+3.9} \mathrm{pb}$. This experiment confirms the discovery of element 111 by the Darmstadt group who used the ${ }^{209} \mathrm{Bi}\left({ }^{64} \mathrm{Ni}, n\right){ }^{272} 111$ reaction.

PACS numbers: 25.70.-z, 25.70.Gh, 23.60.+e, 27.90.+b

Cold nuclear fusion reactions have been successfully used in the production of superheavy elements, most notably in experiments leading to the discovery of elements 107-111 and 
production of element 112 (see summary in [1]). Other laboratories have successfully used these reactions for the production of transactinide elements; e.g., Ref. [2-4]. These reactions use shellstabilized ${ }^{207,208} \mathrm{~Pb}$ and ${ }^{209} \mathrm{Bi}$ targets with projectile energies near the Coulomb barrier to produce compound nuclei with low excitation energies $(\approx 10-16 \mathrm{MeV}[1])$. Only one neutron evaporates from the compound nucleus and the interfering exit channels $2 n, p x n$, $\alpha x n$, etc. are nearly completely eliminated. In the case of elements 110 (darmstadtium, Ds) and 111, a change of target from ${ }^{208} \mathrm{~Pb}$ to ${ }^{209} \mathrm{Bi}$ allows for the production of both using ${ }^{64} \mathrm{Ni}$ projectiles. This additional proton in the target results in a decrease of the maximum $1 n$ cross section from $15_{-6}^{+9} \mathrm{pb}$ [1] to $2.9_{-1.3}^{+1.9} \mathrm{pb}[5]$

Among the goals of our experiments were production of ${ }^{272} 111$ in the odd- $Z$ projectile reaction ${ }_{29}^{65} \mathrm{Cu}+{ }_{82}^{208} \mathrm{~Pb}$, and comparison of the resulting cross section to that obtained using the ${ }_{28}^{64} \mathrm{Ni}+$ ${ }_{83}^{209} \mathrm{Bi}$ reaction. The ${ }^{208} \mathrm{~Pb}\left({ }^{64} \mathrm{Ni}, n\right){ }^{271} \mathrm{Ds}$ excitation function was measured first to aid in the estimation of the optimum ${ }^{65} \mathrm{Cu}$ beam energy for the production of ${ }^{272} 111$. The current work serves as an independent confirmation of the discovery of element 111 [6] using a different reaction. It also demonstrates a rather simple method for estimating optimum projectile energies for production of heavy elements in cold fusion reactions which have very narrow excitation functions. Experiments were carried out at the Lawrence Berkeley National Laboratory (LBNL) 88-Inch Cyclotron using the Berkeley Gas-filled Separator (BGS). The BGS has been described previously [7], although several improvements have been made. The focal plane strip detector now contains 48 vertical strips with an active area $179 \mathrm{~mm}$ wide by $58 \mathrm{~mm}$ high. Horizontal positions are determined by the 3.625-mm wide strips (numbered 0-47 from high to low magnetic rigidity) and vertical positions (from $-29 \mathrm{~mm}$ to $+29 \mathrm{~mm}$ ) are determined by resistive charge division. Thirty-two non-position-sensitive "upstream” detectors are mounted 
perpendicular to the face of the main strip detectors. An event depositing more than $\approx 300 \mathrm{keV}$ in a strip detector or an upstream detector triggers the list-mode data acquisition system. Twelve "punchthrough" detectors mounted directly downstream of the strip detectors provide a veto for light, low-ionizing particles which pass through the strip detectors. All detectors consist of 300$\mu \mathrm{m}$-thick Si. Detectors were calibrated using external $\alpha$-particle sources and products of the ${ }^{40} \mathrm{Ar}+{ }^{208} \mathrm{~Pb}$ and ${ }^{64} \mathrm{Ni},{ }^{65} \mathrm{Cu}+{ }^{116,120} \mathrm{Sn},{ }^{\text {nat }} \mathrm{BaBr}_{2}$ reactions. Energy calibrations for high-energy events were extrapolated from these data. The energy uncertainty $\sigma_{\mathrm{E}}$ was $\approx 20 \mathrm{keV}$ for $\alpha$ particles fully stopped in the focal plane and $\approx 50 \mathrm{keV}$ for $\alpha$ particle events "reconstructed" from the sum of focal plane and upstream detector energies (all uncertainties in this paper are at the $1 \sigma[68 \%]$ confidence level). In general, the position resolution for a single event $\sigma_{p o s}$ was $\approx 2800 E^{-1} \mathrm{keV} \mathrm{mm}$. A multi-wire avalanche counter upstream of the focal plane discriminated implantation events from radioactive decays. An online analysis program using a preliminary calibration initiated a fast beam shutoff ( $\approx 140 \mu$ s) upon detecting a heavy element decay chain. Targets were $470 \pm 60-\mu \mathrm{g} / \mathrm{cm}^{2}\left(98.4 \%{ }^{208} \mathrm{~Pb}, 1.1 \%{ }^{207} \mathrm{~Pb}, 0.5 \%{ }^{206} \mathrm{~Pb}\right)$ deposited on $35-\mu \mathrm{g} / \mathrm{cm}^{2}$ ${ }^{\text {nat }} \mathrm{C}$ backing foils and covered with $5-\mu \mathrm{g} / \mathrm{cm}^{2}{ }^{\text {nat }} \mathrm{C}$. Nine arc-shaped target segments were mounted on the periphery of a 35.6-cm diameter wheel rotating at $450 \mathrm{rpm}$. The beam passed through a $45-\mu \mathrm{g} / \mathrm{cm}^{2}{ }^{\text {nat }} \mathrm{C}$ entrance window (separating the evacuated beamline from the gasfilled separator) and a negligible amount of He gas before striking the target backing. Beam energy losses were calculated using the SRIM-2003 program [8]. Ginter et al. [4] observed two ${ }^{271} \mathrm{Ds}$ decay chains in the ${ }^{208} \mathrm{~Pb}\left({ }^{64} \mathrm{Ni}, n\right)$ reaction at a center-oftarget energy $E_{\text {cot }}$ of $309.2 \mathrm{MeV}$ with a cross section of $8.3_{-5.3}^{+11} \mathrm{pb}$. (All energies in this paper are reported in the laboratory frame unless otherwise specified). In the current experiments, two additional energies were run. In the first, a dose of $2.9 \times 10^{17}{ }^{64} \mathrm{Ni}^{14+}$ projectiles at $319.8 \mathrm{MeV}$ 
was collected during a five-day experiment. At $E_{\text {cot }}=314.3 \mathrm{MeV}$ the calculated center-of-target excitation energy $E_{\text {cot }}^{*}$ was $16.2 \mathrm{MeV}$ using masses from Ref. [9]. The BGS magnets were set to a magnetic rigidity $B \rho$ of $2.04 \mathrm{~T} \mathrm{~m}$, based on results from Ref. [4]. The BGS was filled with He at $0.5-0.9$ torr. In the second, a dose of $2.3 \times 10^{17}{ }^{64} \mathrm{Ni}^{14+}$ projectiles at $317.0 \mathrm{MeV}$ was collected in five days of beam time, with $E_{\text {cot }}=311.5 \mathrm{MeV}$ and $E_{\text {cot }}^{*}=14.1 \mathrm{MeV}$. Based on the results of the first run, the BGS magnets were readjusted to $B \rho=2.06 \mathrm{~T} \mathrm{~m}$.

Correlated EVR- $\alpha$ and $\alpha-\alpha$ searches were conducted offline using two independent analysis codes. Decay chains attributed to ${ }^{271}$ Ds are shown in Fig. 1(a). Chains $1-2$ were observed at $E_{\text {cot }}$ $=314.3 \mathrm{MeV}$ and chains 3-7 were observed at $E_{\mathrm{cot}}=311.5 \mathrm{MeV}$. The average observed EVR implantation pulse height corresponded to $25.9 \pm 1.6 \mathrm{MeV}$. The observed energies have not been corrected for pulse-height defect. In all cases except chain 4 [10], the decay sequence could be followed to the decay of ${ }^{255} \mathrm{No}\left(t_{1 / 2}=3.1 \mathrm{~min}\right)$.

The data are in excellent agreement with previously reported ${ }^{271}$ Ds decay data $[1-2,4]$. The $\alpha$ events assigned to ${ }^{271}$ Ds in chains 5-7 have an average energy of $10753 \mathrm{keV}$ and are assigned to the known transition at $10738 \mathrm{keV}$. The 10688-keV event in chain 1 can be assigned to either the transition at $10681 \mathrm{keV}$ or the one at $10738 \mathrm{keV}$ within reconstructed $\alpha$ event error. The half-life of the six ${ }^{271}$ Ds events (chains 1-3, 5-7) was calculated to be $1.6_{-0.5}^{+0.9} \mathrm{~ms}$. (The MLDS code [12] was used to calculate half-lives and uncertainties). This result is in excellent agreement with the reported half-life of $1.63_{-0.29}^{+0.44} \mathrm{~ms}[2]$.

Only online analysis results are available [10] for chain 4. An implantation event was followed by two high-energy $\alpha$ events (9880 keV and $9227 \mathrm{keV}$ ) that can be assigned to the decays of ${ }^{267} \mathrm{Hs}$ and ${ }^{263 \mathrm{~m}} \mathrm{Sg}$, respectively. The remaining $\alpha$ particles in this chain may have been emitted 
out of the front of the focal plane ( $\approx 45 \%$ probability each) and were not recorded by the online analysis. The 22-ms lifetime reported with ${ }^{267} \mathrm{Hs}$ in chain 4 is the sum of the ${ }^{271} \mathrm{Ds}$ and ${ }^{267} \mathrm{Hs}$ lifetimes as reported by the online analysis.

The ${ }^{267}$ Hs events from chains 1 , 4, and 5 with an average energy of $9877 \mathrm{keV}$ are consistent with the known 9882-keV decay. The other full-energy $\alpha$ event in chain 2 (9830 keV) can be assigned to the $9829-\mathrm{keV}$ transition. The observation of two unusually short ${ }^{267} \mathrm{Hs}$ lifetimes of $0.482 \mathrm{~ms}$ and $2.45 \mathrm{~ms}$ in chains 2 and 7, respectively, suggests the presence of a short-lived isomer with half-life $0.94_{-4.5}^{+12} \mathrm{~ms}$. The other ${ }^{267} \mathrm{Hs}$ lifetimes (chains $1,3,5$, and 6) are consistent with a half-life of $55_{-18}^{+32} \mathrm{~ms}$, in good agreement with the previously reported ${ }^{267} \mathrm{Hs}$ half-lives of $59_{-15}^{+30} \mathrm{~ms}[1]$ and $53 \mathrm{~ms}[2]$.

The six full-energy ${ }^{263} \mathrm{Sg}$ events in chains 1-6 have an average energy of $9245 \mathrm{keV}$ and are assigned to ${ }^{263} \mathrm{Sg}^{\mathrm{m}}\left(E_{\alpha}=9248 \mathrm{keV}\right)$ [11]. The half-life of these six events is $290_{-90}^{+170} \mathrm{~ms}$, in excellent agreement with the known ${ }^{263} \mathrm{Sg}^{\mathrm{m}}$ half-life $\left(310_{-80}^{+160} \mathrm{~ms}\right)$. The lifetime of the escape event in chain 7 (270 ms) is consistent with both the ${ }^{263} \mathrm{Sg}^{\mathrm{m}}$ and ${ }^{263} \mathrm{Sg}^{\mathrm{g}}\left(\mathrm{t}_{1 / 2}=800 \pm 200 \mathrm{~ms}\right)$ halflives, so no definitive assignment is possible.

The ${ }^{259} \mathrm{Rf}$ events from chains 5 (8898 keV) and 7 (8908 keV) clearly belong to the known ${ }^{259} \mathrm{Rf}$ transition at $8895 \mathrm{keV}$. The chain 2 event (8863 keV) can be assigned to the transition at 8861 keV. Within uncertainties, the reconstructed $\alpha$ event in chain 3 (8792 keV) can be assigned to the transition at $8756 \mathrm{keV}$, and the completely stopped $\alpha$ event from chain 1 (8878 $\mathrm{keV}$ ) can be assigned to either of the 8861 - and $8895-\mathrm{keV}$ transitions. The measured half-life of all six ${ }^{259} \mathrm{Rf}$ events is $2.2_{-0.8}^{+1.7} \mathrm{~s}$, compared to the previously reported values $2.6 \mathrm{~s}$ [2] and $2.6_{-0.7}^{+1.4} \mathrm{~s}$ [1]. 
The overall count rates of $\alpha$ particles (fully stopped and reconstructed) at full beam intensity in a 3.0-mm pixel from $8500-12000 \mathrm{keV}$ in the $E_{\mathrm{cot}}=314.3 \mathrm{MeV}$ and $E_{\mathrm{cot}}=311.5 \mathrm{MeV}$ runs were $7.8 \times 10^{-6} \mathrm{~Hz}$ and $1.7 \times 10^{-5} \mathrm{~Hz}$, respectively. These rates were used to estimate the Poisson probability of observing exactly two $\alpha$ events within $15 \mathrm{~s}$ of an implantation event, since all ${ }^{271}$ Ds decay chains had at least two fully-stopped $\alpha$ particles among the first four members of the chain. Multiplying the number of implantation events and the Poisson probabilities, the expected numbers of random EVR- $\alpha-\alpha$ correlations were $6.2 \times 10^{-4}$ and $5.6 \times 10^{-3}$, respectively. These calculations give upper limits because there are no requirements that the observed events agree with previous data or that additional escape $\alpha$ particles be present to complete the decay chain.

Six events observed in these decay chains were assigned to ${ }^{255}$ No based on its known $\alpha$ decay properties. The observed half-life of all six events was $200_{-70}^{+140} \mathrm{~s}$, compared to $190 \pm 10 \mathrm{~s}$ measured previously [11]. An analysis similar to the one above indicates that each ${ }^{255}$ No event has a probability of $\approx 5 \%$ of resulting from a random correlation.

The observed cross sections at $E_{\text {cot }}=314.3 \mathrm{MeV}\left(E_{\text {cot }}^{*}=16.2 \mathrm{MeV}\right)$ and $E_{\text {cot }}=311.5 \mathrm{MeV}\left(E_{\text {cot }}^{*}\right.$ $=14.1 \mathrm{MeV}$ ) are $7.7_{-5.2}^{+10} \mathrm{pb}$ and $20_{-11}^{+15} \mathrm{pb}$, respectively. Uncertainties in cross sections are computed according to methods described in Ref. [13]. Chain 3 was obtained with a set of targets that was too thick for an accurate cross section measurement and is not included in cross section calculations. Its observed lifetimes and decay energies have been used in the appropriate discussions. Combined with previous results [4], the current work establishes an excitation function for the ${ }^{208} \mathrm{~Pb}\left({ }^{64} \mathrm{Ni}, n\right){ }^{271} \mathrm{Ds}$ reaction at the LBNL 88-Inch Cyclotron. The average magnetic rigidity of all seven ${ }^{271}$ Ds EVRs was $B \rho=2.09 \pm 0.03 \mathrm{~T} \mathrm{~m}$. 
Using Ref. [14-15] an estimate was made of the optimum ${ }^{65} \mathrm{Cu}+{ }^{208} \mathrm{~Pb}$ bombarding energy from the optimum ${ }^{64} \mathrm{Ni}+{ }^{208} \mathrm{~Pb}$ bombarding energy. These references suggest that the maximum $1 n$ cross section is obtained $\approx 0.3 \mathrm{MeV}$ above the threshold for second-chance fission. It was estimated [15] that the maximum cross section of the ${ }^{208} \mathrm{~Pb}\left({ }^{65} \mathrm{Cu}, n\right){ }^{272} 111$ reaction should be obtained at $E_{\text {cot }} 9.6 \mathrm{MeV}$ higher than in the ${ }^{208} \mathrm{~Pb}\left({ }^{64} \mathrm{Ni}, n\right){ }^{271} \mathrm{Ds}$ reaction. Thus, a new experiment was performed with a ${ }^{65} \mathrm{Cu}$ beam at $E_{\mathrm{cot}}=321.1 \mathrm{MeV}\left(E_{\mathrm{cot}}^{*}=13.2 \mathrm{MeV}\right)$. The beam energy out of the cyclotron was $326.9 \mathrm{MeV}$. The same targets and experimental conditions were used in this experiment as in the two ${ }^{271}$ Ds runs. The magnetic rigidity of ${ }^{272} 111$ in this reaction was estimated to be $2.08 \mathrm{~T} \mathrm{~m}$. During two five-day runs, a total dose of $6.6 \times 10^{17}{ }^{65} \mathrm{Cu}^{15+}$ particles was collected.

One decay chain of interest was observed in the experiment and is shown in Fig. 1(b). An implantation event with a pulse height corresponding to $28.58 \mathrm{MeV}$ was followed 0.263 ms later by an $\alpha$ event with energy $11042 \mathrm{keV}$. This energy agrees well with the $11027-\mathrm{keV}^{272} 111 \alpha$ energy group reported in Ref. [5] and the main $\alpha$ group at $\approx 11000 \mathrm{keV}$ in Fig. 4(b) of Ref. [2]. The observed lifetime was $0.263 \mathrm{~ms}$, compared to the reported half-lives of $1.6_{-0.5}^{+1.1} \mathrm{~ms}$ [5] and $3.8_{-0.8}^{+1.4} \mathrm{~ms}$ [2]. A subsequent $10114-\mathrm{keV} \alpha$ decay is consistent with the $10097-\mathrm{keV}^{268} \mathrm{Mt}$ event observed in chain 2 of Ref. [6] within uncertainty and the observed 12.6-ms lifetime agrees with the reported ${ }^{268} \mathrm{Mt}$ half-lives of $42_{-12}^{+29} \mathrm{~ms}$ [5] and $21_{-5}^{+8} \mathrm{~ms}$ [2]. These three signals, correlated in energy, time, and position, provide evidence of the presence of a high- $Z$ element, which we assign to ${ }^{272} 111$ based on the similarity of the observed decay properties to those observed previously by other groups.

Additionally, three more $\alpha$-like signals were observed following the ${ }^{268} \mathrm{Mt}$ event. An escape $\alpha$ is assigned to ${ }^{264} \mathrm{Bh}$, as the observed lifetime of 1.16 s agrees well with the reported ${ }^{264} \mathrm{Bh}$ half- 
lives of $1.0_{-0.3}^{+0.7} \mathrm{~s}$ [5] and $0.9_{-0.2}^{+0.3} \mathrm{~s}$ [2]. A reconstructed $\alpha$ event, with sum energy $9416 \mathrm{keV}$, is assigned to the decay of ${ }^{260} \mathrm{Db}$ as its relatively high decay energy is consistent with the $\alpha$ group at $\approx 9300 \mathrm{keV}$ shown in Fig. 4(b) of Ref. [2]. The observed lifetime (1.45 s) is consistent with the previously reported ${ }^{260} \mathrm{Db}$ half-life $(1.52 \pm 0.13 \mathrm{~s}[11])$. Within the detector resolution, a second reconstructed $\alpha$ with energy $8613 \mathrm{keV}$ and lifetime $3.16 \mathrm{~s}$ can be assigned to the known ${ }^{256} \operatorname{Lr}\left(t_{1 / 2}=28 \pm 3 \mathrm{~s}\right)$ transition at $8635 \mathrm{keV}$ [11]. No other $\alpha$ events with energy greater than $1000 \mathrm{keV}$ or fission events were observed at any location in the strip within $150 \mathrm{~s}$ of the implantation event.

The observed ${ }^{272} 111$ EVR magnetic rigidity in He was $B \rho=2.10 \mathrm{~T} \mathrm{~m}$. The same random correlation analysis was performed as for the ${ }^{271}$ Ds experiments. The beam did not shut off during this event (see Fig. 1) because of a bias supply problem in the beam "chopper," so the calculation must be done with count rates at maximum beam intensity. The average count rate of $\alpha$ particles (fully stopped and reconstructed) in both runs with energies $8200-12000 \mathrm{keV}$ in a 3.0-mm pixel was $4.8 \times 10^{-6} \mathrm{~Hz}$. Based on the number of implantation events and the Poisson probability of observing two or four $\alpha$ events within $180 \mathrm{~s}$ of an implantation event at the above count rates, the expected numbers of random EVR- $\alpha-\alpha$ and EVR- $\alpha-\alpha-\alpha-\alpha$ correlations due to unrelated events are 0.14 and $8.5 \times 10^{-9}$, respectively Based on the observed decay chain, a cross section of $1.7_{-1.4}^{+3.9} \mathrm{pb}$ was calculated for the ${ }^{208} \mathrm{~Pb}\left({ }^{65} \mathrm{Cu}, n\right){ }^{272} 111$ reaction. In agreement with predictions [15], it appears to be somewhat lower than the cross section maxima of $2.9_{-1.3}^{+1.9} \mathrm{pb}$ [5] and $2.6_{-1.5}^{+2.3} \mathrm{pb}$ [2] reported for the ${ }^{209} \mathrm{Bi}\left({ }^{64} \mathrm{Ni}, n\right){ }^{272} 111$ reaction.

In conclusion, the cross section of the ${ }^{208} \mathrm{~Pb}\left({ }^{65} \mathrm{Cu}, n\right){ }^{272} 111$ reaction has been measured for the first time and is comparable to that for the ${ }^{209} \mathrm{Bi}\left({ }^{64} \mathrm{Ni}, n\right){ }^{272} 111$ reaction. Our work provides an 
independent confirmation of the discovery of element 111 [6] in a new reaction. Such confirmation experiments are critical in establishing the validity of claims for the discovery of new elements.

We thank D. Leitner and the staff of the LBNL 88-Inch Cyclotron for developing and providing intense, stable beams of ${ }^{64} \mathrm{Ni}$ and ${ }^{65} \mathrm{Cu}$. The authors wish to express their appreciation to W. J. Swiatecki for many stimulating and informative discussions. This work was supported in part by the Office of High Energy and Nuclear Physics, Nuclear Physics Division, United States Department of Energy under contract number DE-AC03-76SF00098. 


\section{REFERENCES AND FOOTNOTES}

[1] S. Hofmann, Rep. Prog. Phys. 61, 639 (1998).

[2] K. Morita et al., Nucl. Phys. A734, 101 (2004).

[3] S. Grévy et al., J. Nucl. Radiochem. Sci. 3, 9 (2002).

[4] T. N. Ginter et al., Phys. Rev. C 67, 064609 (2003).

[5] S. Hofmann et al., Eur. Phys. J. A 14, 147 (2002).

[6] S. Hofmann et al., Z. Phys. A 350, 281 (1995).

[7] K. E. Gregorich and V. Ninov, J. Nucl. Radiochem. Sci. 1, 1 (2000).

[8] J. F. Ziegler, computer code SRIM-2003, available from http://www.srim.org.

[9] W. D. Myers and W. J. Swiatecki, Nucl. Phys. A601, 141 (1996).

[10] As intended, our data acquisition system periodically closed the current data file and opened a new one. A previously undetected design flaw allowed the data from chain 4 to be discarded because it could not be written to disk while the system was changing files. The manufacturer has supplied us with corrected software.

[11] R. B. Firestone, Table of Isotopes, (John Wiley \& Sons, New York, 1996), 8th ed.

[12] K. E. Gregorich, Nucl. Instrum. Methods Phys. Res. A302, 135 (1991).

[13] K.-H. Schmidt, C.-C. Sahm, K. Pielenz, and H.-G. Clerc, Z. Physik A 316, 19 (1984).

[14] W. J. Swiatecki, K. Siwek-Wilczynska, and J. Wilczynski, Acta Phys. Pol. B, 34, 2049 (2003).

[15] W. J. Swiatecki (private communication); W. J. Swiatecki, K. Siwek-Wilczynska, and J. Wilczynski, Phys. Rev. C (to be submitted). 


\section{FIGURE CAPTION}

FIG. 1. Decay chains observed in the (a) ${ }^{208} \mathrm{~Pb}\left({ }^{64} \mathrm{Ni}, n\right){ }^{271} \mathrm{Ds}$ and (b) ${ }^{208} \mathrm{~Pb}\left({ }^{65} \mathrm{Cu}, n\right){ }^{272} 111$ reactions. Implantation event is shown above each chain. Notation $x+y=z$ indicates that strip and upstream detector signals were observed simultaneously with energies $x$ and $y$, respectively, and sum $z$ (all in keV). Black triangle in lower right corner of box indicates beam was shut off. Events with energies and positions estimated from one available strip signal only are denoted by $(\boldsymbol{\Phi})$. Lifetime marked with diamond $(\bullet)$ is sum of ${ }^{271}$ Ds and ${ }^{267}$ Hs lifetimes. 


\section{FIGURE}

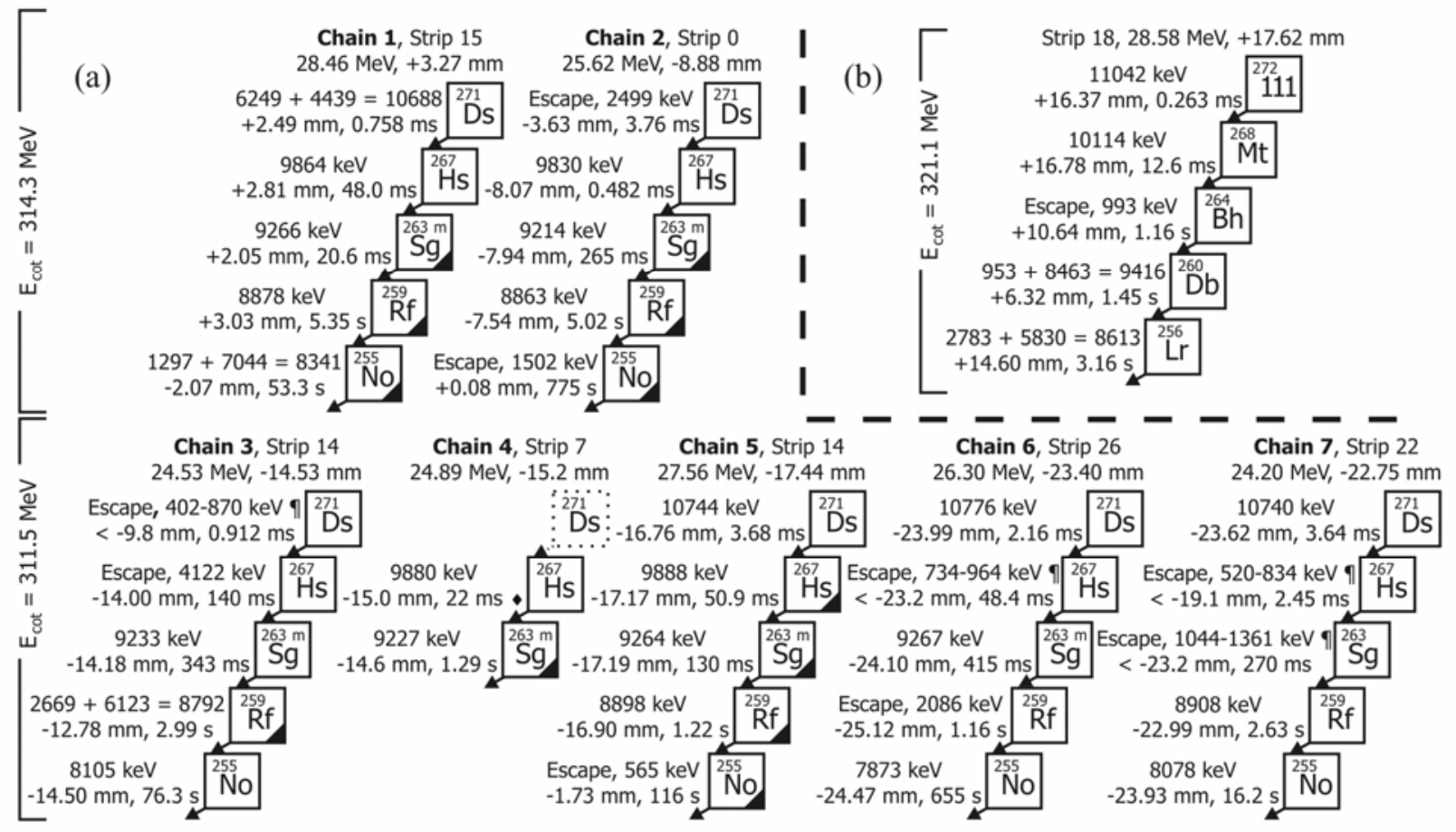

FIG. 1. 